\title{
Efeitos da mobilização neural sobre a dor, força muscular e amplitude de movimento: revisão de literatura
}

\author{
Effects of neural mobilization about pain, muscular \\ strength and movement amplitude: literature review
}

Ana Quesia Pereira Castelo BRANCO

Instituto de Ensino Superior Blauro Cardoso de Mattos (FaSerra). Manaus/AM, Brasil
RESUMo A mobilização neural é uma modalidade de tratamento utilizada desde 1800, com o objetivo de restaurar o movimento e a elasticidade do sistema nervoso por meio de técnicas de tensão e alongamento, restabelecendo a neurodinâmica adequada e diminuindo os sintomas gerados por lesões compressivas nervosas. O objetivo deste estudo foi revisar a literatura brasileira existente acerca do uso da mobilização neural e seus efeitos sobre a dor, força muscular e amplitude de movimento nas diversas disfunções nervosas. Realizou-se um levantamento bibliográfico dos artigos relacionados à mobilização neural publicados entre 2010 e 2018, em língua portuguesa, nas bases de dados SciELO e LILACS, a fim de analisar os efeitos da técnica em indivíduos sadios e portadores de patologias compressivas. A técnica demonstrou resultados positivos na diminuição da dor, aumento da força muscular e amplitude de movimento na população avaliada. Conclui-se que a mobilização neural é eficaz ao ser usada para reabilitar a função motora e reduzir a dor, porém, devido ao baixo número de estudos a respeito da técnica, sugere-se que sejam feitas mais pesquisas, para que haja um maior embasamento científico sobre os seus efeitos.

Palavras-Chave: Sistema Nervoso. Axonotmese. Neurotmese.

Abstract Neural mobilization is a treatment modality used since 1800 , with the objective of restoring the movement and elasticity of the nervous system through tension and stretching techniques, restoring adequate neurodynamics and reducing the symptoms generated by nerve compressive lesions. The objective of this study was to review the brazilian literature on the use of neural mobilization and its effects on pain, muscle strength and movement amplitude in various nervous dysfunctions. A literature review of articles related to neural mobilization, published between 2010 and 2018, in the Portuguese language, in the SciELO and LILACS databases, was carried out in order to analyze the effects of the technique on healthy individuals and patients with compressive pathologies. The technique demonstrated positive results in pain reduction, increased muscle strength and range of motion in the evaluated population. It is concluded that neural mobilization is effective when it is used to rehabilitate motor function and reduce pain, but due to the low number of studies about the technique, it is suggested that more research be done to provide a more scientific basis on the effects. Keywords: Nervous System. Axonotmesis. Neurotmesis. 


\section{INTRODUÇÃO}

A principal função do Sistema Nervoso (SN) é a condução de impulsos; porém, esta é extremamente dependente da parte mecânica desse sistema, e vice-versa. A interligação da função mecânica e fisiológica do SN foi reunida no termo neurodinâmica. Se a função desses sistemas apresenta normalidade neurodinâmica, significa que suas propriedades mecânicas e fisiológicas estão normais. ${ }^{1}$

Quando a neurodinâmica está alterada, ocorre o que se denomina de Tensão Neural Adversa (TNA), que consiste numa resposta mecânica e fisiológica anormal quando a amplitude normal do SN e sua capacidade de alongamento são testadas. ${ }^{2}$

O SN se adapta à mobilidade corporal por meio de movimentos relativos às estruturas que o envolvem. Por ser um tecido contínuo, possui propriedades elásticas, podendo encurtar-se ou se alongar em resposta a movimentos dos segmentos corporais. Também se adapta aos movimentos pelas suas propriedades mecânicas (tensão), ao mesmo tempo em que realiza sua principal função: a condução de impulsos. ${ }^{3}$ A atividade adequada do SN depende de sua integridade. O comprometimento da mecânica e da fisiologia do SN pode resultar em outras disfunções próprias do mesmo, bem como, das estruturas musculoesqueléticas que recebem a sua inervação. ${ }^{1}$

Os nervos periféricos são alvos constantes de lesões por esmagamento, compressão e transecção. Tais lesões resultam em dor, redução ou perda da sensibilidade e motricidade na área inervada. ${ }^{4}$ Essas alterações provocam um impacto importante sobre o paciente e sua capacidade de realizar ati- vidades básicas de vida diária, bem como, o retorno ao trabalho, tornando-se de relevância social e econômica. ${ }^{5}$

A Mobilização Neural (MN) tem como objetivo restaurar o movimento e a elasticidade do $\mathrm{SN}$, o que promove o retorno às suas funções normais. ${ }^{3}$ A técnica vem sendo utilizada desde 1800, sendo progressivamente aperfeiçoada tanto na teoria quanto na prática. ${ }^{6}$

A MN apresenta um conjunto de técnicas que propõem a tensão do neuroeixo e o alongamento da estrutura por meio de movimentos adequados associados à aplicação de uma força mecânica, que induz o ajuste do sistema nervoso, permitindo que estruturas interligadas sejam reestabelecidas e se recuperem quanto à funcionalidade e extensibilidade, favorecendo a neurodinâmica adequada. $^{7}$

Este estudo teve como objetivo revisar a literatura brasileira existente a respeito da utilização da mobilização neural e suas repercussões sobre a dor, força muscular e amplitude de movimento nas diversas disfunções nervosas.

\section{Metodologia}

Realizou-se um levantamento bibliográfico de artigos científicos relacionados à mobilização neural nas bases de dados SciELO (Scientific Electronic Library Online) e LILACS (Literatura Latino-Americana e do Caribe em Ciências da Saúde), durante os meses de junho e julho de 2018. Para a pesquisa dos artigos, foram utilizadas as palavras-chave associadas: "mobilização neural; dor" "mobilização neural; força muscular", "mobilização neural; amplitude de movimento". 
Os artigos foram filtrados por idioma e ano de publicação, sendo considerados estudos apenas em língua portuguesa, publicados entre o período de 2010 a 2018, no formato de artigo original, com texto completo disponível gratuitamente, sendo excluídos ainda os artigos duplicados. Foram selecionados artigos que abordaram a mobilização neural como forma de intervenção tanto em seres humanos quanto em animais com patologias nervosas compressivas. Os dados foram organizados por meio de fluxograma, tabulados no Microsoft Office Word e analisados descritivamente.

\section{RESUltados}

Inicialmente, foram encontrados 29 artigos, sendo nove artigos na base de dados SciELO e 20 artigos no LILACS. Desses, apenas seis se adequaram aos critérios de inclusão. A Figura 1 mostra o fluxograma dos artigos encontrados e selecionados para esta revisão. Os principais artigos que utilizaram a mobilização neural como forma de intervenção se encontram descritos na Tabela 1.

Figura 1. Fluxograma de busca e seleção dos artigos para revisão.

\section{Identificados: 29 artigos}

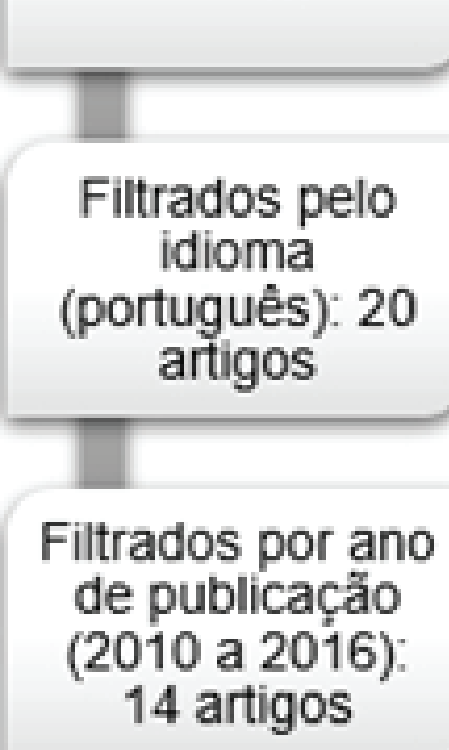

\section{Duplicados: 8 artigos}


Tabela 1. Principais artigos selecionados para revisão.

\begin{tabular}{|c|c|c|c|c|}
\hline AUTOR/ANO & OBJETIVO & DELINEAMENTO & PATOLOGIA & RESULTADO \\
\hline $\begin{array}{c}\text { Lopes RSD, Barja } \\
\text { PR, Matos LKBL, } \\
\text { Delmondes FF, Silva } \\
\text { KAS, Lima MO (2010) }\end{array}$ & $\begin{array}{l}\text { Comparar a influência } \\
\text { do alongamento } \\
\text { muscular e da MN na } \\
\text { força de quadríceps. }\end{array}$ & $\begin{array}{l}39 \text { voluntários foram } \\
\text { divididos em três } \\
\text { grupos. Um grupo } \\
\text { foi submetido ao } \\
\text { alongamento estático } \\
\text { de } 30 \text { segundos, outro } \\
\text { grupo foi submetido à } \\
\text { MN, e o terceiro, um } \\
\text { grupo controle. }\end{array}$ & - & $\begin{array}{l}\text { Melhora significativa } \\
\text { na força muscular do } \\
\text { grupo submetido à MN. }\end{array}$ \\
\hline $\begin{array}{l}\text { Lima KCS, Piauilino } \\
\text { PMM, Franco RM, } \\
\text { Silva RSDL (2016) }\end{array}$ & $\begin{array}{l}\text { Analisar e comparar os } \\
\text { efeitos do alongamento } \\
\text { muscular, da } \mathrm{MN} \mathrm{e} \\
\text { estimulação vibratória. }\end{array}$ & $\begin{array}{l}40 \text { indivíduos } \\
\text { foram divididos em } \\
\text { quatro grupos. G1: } \\
\text { realizou MN, G2: } \\
\text { alongamento muscular, } \\
\text { G3: submetido à } \\
\text { estimulação vibratória, } \\
\text { e G4: um grupo } \\
\text { controle. Os grupos } \\
\text { foram avaliados pela } \\
\text { Escala de Berg e Timed } \\
\text { Get Up And Go. }\end{array}$ & $\begin{array}{l}\text { Acidente } \\
\text { Vascular } \\
\text { Encefálico. }\end{array}$ & $\begin{array}{l}\text { Melhora significativa } \\
\text { no equilíbrio e } \\
\text { mobilidade funcional. } \\
\text { Os melhores resultados } \\
\text { surgiram após a MN e } \\
\text { estimulação vibratória. }\end{array}$ \\
\hline $\begin{array}{c}\text { Barbosa APB, Leal SS } \\
(2015)\end{array}$ & $\begin{array}{l}\text { Avaliar o efeito } \\
\text { imediato da } \\
\text { mobilização do nervo } \\
\text { ciático sobre o ganho } \\
\text { de amplitude de } \\
\text { movimento de flexão de } \\
\text { quadril em indivíduos } \\
\text { assintomáticos. }\end{array}$ & $\begin{array}{c}50 \text { indivíduos } \\
\text { assintomáticos foram } \\
\text { divididos em } 2 \\
\text { grupos. No grupo } 1 \\
\text { foi realizada avaliação } \\
\text { da ADM, mobilização } \\
\text { neural e reavaliação do } \\
\text { MID. No grupo } 2 \text { foi } \\
\text { feita avaliação do MID, } \\
\text { mobilização neural no } \\
\text { MIE e reavaliação do } \\
\text { MID. }\end{array}$ & - & $\begin{array}{l}\text { Aumento na média } \\
\text { de ADM nos dois } \\
\text { grupos, com melhores } \\
\text { resultados para o } \\
\text { grupo submetido à } \\
\text { mobilização indireta. }\end{array}$ \\
\hline $\begin{array}{l}\text { Karvat J, Antunes } \\
\text { JS, Bernardino GR, } \\
\text { Kakihata CMM, } \\
\text { Bertolini GRF (2014) }\end{array}$ & $\begin{array}{l}\text { Verificar o efeito } \\
\text { do LASER de baixa } \\
\text { intensidade associado à } \\
\text { MN na ciatalgia. }\end{array}$ & $\begin{array}{l}28 \text { ratas Wistar foram } \\
\text { divididas em } 4 \text { grupos. } \\
\text { G1: placebo, G2: MN, } \\
\text { G3: LASER, G4: MN } \\
\text { e LASER. As ratas } \\
\text { foram submetidas } \\
\text { ao tratamento após } \\
\text { cirurgia de compressão } \\
\text { do nervo ciático, e } \\
\text { avaliadas antes da lesão } \\
\text { nervosa e no } 3^{\circ} .4^{\circ} . \text {, } \\
7^{\circ} ., 10^{\circ} ., 14^{\circ} . \text { e } 15^{\circ} . \text { dias } \\
\text { de PO. }\end{array}$ & Ciatalgia & $\begin{array}{c}\text { Não houve diferença } \\
\text { significativa entre os } \\
\text { grupos. }\end{array}$ \\
\hline
\end{tabular}

Legenda: $\mathrm{MN}=$ mobilização neural; $\mathrm{ADM}=$ amplitude de movimento; $\mathrm{MID}=$ membro inferior direito; $\mathrm{MIE}=$ membro inferior esquerdo; $\mathrm{PO}=$ pós-operatório. 


\section{Discussão}

A análise dos artigos selecionados apontou que a mobilização neural demonstra resultados positivos na redução da dor, no ganho de força muscular e ganho de amplitude de movimento em indivíduos com lesões nervosas. Apesar dos bons resultados da utilização da técnica na prática clínica, ainda são poucos os estudos que abordam os seus efeitos. ${ }^{8}$

O conceito e as técnicas da mobilização neural não são recentes. Uma forma de tratamento cirúrgico conhecido como "estiramento nervoso" foi muito utilizada no fim do século passado na França e Inglaterra. A técnica geralmente era aplicada em lesões do nervo ciático e plexo braquial, e foi usada para uma variedade de queixas. ${ }^{9}$

Os nervos são móveis e capazes de distensão considerável devido ao seu arranjo, contudo estão suscetíveis a vários tipos de lesão como compressão, laceração e tensão excessiva. Seddon classifica as lesões nervosas com base nas alterações estruturais e funcionais ocorridas no nervo com diferentes graus de lesão. A neuropraxia é considerada como o tipo mais brando de lesão, onde ocorre desmielinização segmentar com recuperação geralmente completa, sendo causada por compressão, levando a ligeira isquemia. A axonotmese se caracteriza por lesão axonal, mantendo as coberturas de tecido conjuntivo intactas, sendo causada por compressão prolongada, levando a infarto e necrose. Sua recuperação pode necessitar intervenção cirúrgica. A neurotmese tem como característica o rompimento total do nervo e das coberturas de tecido conjuntivo, em que não é possível recuperar a fibra nervosa sem intervenção cirúrgica. É causada principalmente por ferimentos por arma de fogo ou cortantes. ${ }^{10}$

No decorrer dos últimos anos, observou-se um grande avanço na utilização da mobilização neural. ${ }^{11}$ Uma vez decidido usar a técnica, o tratamento pode ser escolhido baseando-se em duas considerações principais: o conceito de Maitland, ou seja, o tratamento dos sinais e sintomas ancorados na gravidade, irritabilidade e natureza da desordem, e o desenvolvimento de ideias sobre a patologia, como o local da mecânica alterado, os tecidos neurais envolvidos e estruturas adjacentes que podem interferir na mecânica normal do sistema nervoso. ${ }^{9}$

Os estudos analisados apresentaram controvérsias nos resultados. Lopes ${ }^{11} \mathrm{com}$ parou os efeitos da mobilização neural e do alongamento estático sobre a força muscular de quadríceps, e constatou que, enquanto a mobilização neural auxiliou no aumento de força do músculo, o alongamento causou a diminuição da mesma. O estudo de Barbosa $^{2}$ utilizou a mobilização neural em indivíduos assintomáticos e verificou que técnica é uma alternativa eficaz para o ganho de amplitude de movimento de flexão de quadril.

Um estudo realizado com 29 indivíduos portadores de hanseníase submetidos à mobilização neural durante seis semanas concluiu que a técnica foi capaz de reduzir os níveis de dor de pacientes com lesão do nervo fibular comum, com base na Escala Visual Analógica (EVA). Conforme a escala, os indivíduos submetidos à técnica relataram redução de dor do nível 5 para o nível $2 .{ }^{12} \mathrm{Em}$ contrapartida, Karvat ${ }^{4}$ mostrou que o uso da 
mobilização neural associado ao LASER de baixa intensidade não foi eficaz na redução da dor em ratas Wistar com ciatalgia.

A recuperação de lesões nervosas começa a partir dos sinais de reinervação apresentados pelo paciente, como a contração muscular voluntária e hipersensibilidade. A partir daí, deve-se iniciar a reeducação motora, dessensibilização e a reeducação sensorial discriminativa, na qual o cérebro deve ser retreinado a identificar estímulos após a diminuição da hipersensibilidade. A instrução do fisioterapeuta ao paciente para retomar as atividades diárias é de extrema importância, pois ao diminuir ou evitar as atividades agravantes, por consequência, serão prevenidas novas lesões. ${ }^{10}$

\section{CONSIDERAÇõeS FINAIS}

A mobilização neural se mostrou uma técnica eficaz na diminuição da dor, além de promover o aumento da amplitude de movimento e o ganho de força muscular. Entretanto, devido à escassez de estudos que comprovem a eficácia dessa técnica em humanos, conclui-se que existe a necessidade de se realizar mais pesquisas na área, a fim de que os profissionais que a utilizam tenham maior embasamento científico e segurança ao usá-la em seus pacientes.

\section{REFERÊNCIAS}

1. Vasconcelos DA, Lins LCRF, Dantas EHM. Avaliação da mobilização neural sobre o ganho de amplitude de movimento. Fisioter. Mov., Curitiba, v. 24, n. 4, p. 665-672, out./dez. 2011.

2. Barbosa APB, Leal SS. Análise da eficácia da mobilização neural do nervo isquiático sobre o ganho de ADM. ConScientiae Saúde, 2015; 14 (3): 463-469.

3. Junior HFO, Teixeira AH. Mobilização do sistema nervoso: avaliação e tratamento. Fisioter. Mov., Curitiba, v. 20, n. 3, p. 41-53, jul./set. 2007.

4. Karvat J., Antunes JS, Bernardino GR, Kakihata CMM, Bertolini GRF. Efeito do LASER de baixa potência, da mobilização neural e da sua associação sobre o limiar nociceptivo em ciatalgia experimental. Rev. Dor. São Paulo, 2014, jul./set., 15 (3): 207-10.

5. Bernardino GR, Pelissari D., Paretti AL, Kakihata CMM, Baretta V., Malanotte JÁ et al. Mobilização neural aplicada em diferentes locais sobre a compressão do nervo isquiático de ratos Wistar. ConScientiae Saúde, 2016, 15 (2): 258-265.

6. Andrade EM, Almeida JG. Mobilização neural: tratamento de distúrbios musculoesqueléticos, 2014, abr. [citado 07/07/2018]. Disponível em: www.portalbiocursos.com.br/ohs/data/ docs/32/112_MobilizaYYo_Neural_tratamento_de_distYrbios_musculoesquelYticos.pdf

7. Machado AF, Silva JS, Ferreira ASA, Micheletti JK, Martini FAN. Efeitos imediatos e tardios da mobilização neural sobre força de preensão palmar e complacência neural de membro superior: um ensaio clínico randomizado. ConScientiae Saúde, 2015, 14 (3): 370-377.

8. Araújo BF, Nascimento CM, Busarello FO, Moreira NB, Baroni MP, Carvalho AR et al. Avaliação da força de preensão palmar frente à terapia com mobilização neural. Rev Bras Med Esporte, v. 18, n. 4, jul./ago., 2012.

9. Butler DS, Jones MA. Mobilização do sistema nervoso. Barueri: Manole, 2003.

10. Kisner C., Colby LA. Exercícios terapêuticos - fundamentos e técnicas, 5. ed. São Paulo: Manole, 2009. 
11. Lopes RSD, Barja PR, Matos LKBL, Delmondes FF, Lopes PFD, Silva KAS, et al. Influência do alongamento muscular e da mobilização neural sobre a força do músculo quadríceps. ConScientiae Saúde, 2010; 9 (4): 603-609.

12. Véras LST, Vale RGS, Mello DB, Castro JAF, Dantas EHM. Avaliação da dor em portadores de hanseníase submetidos à mobilização neural. Fisioterapia e Pesquisa, São Paulo, v. 18, n. 1, p. 31-6, jan./mar. 2011.

\section{DAdos DA AUTORA}

\section{Ana Quesia Pereira Castelo Branco}

Graduada em Fisioterapia pela Universidade Paulista (UNIP). Pós-graduada em Fisioterapia Neurofuncional pelo Instituto de Ensino Superior Blauro Cardoso de Mattos (FaSerra). Manaus/AM, Brasil. anacbranco@hotmail.com

Submetido em: 17-9-2018

Aceito em: 21-11-2019 